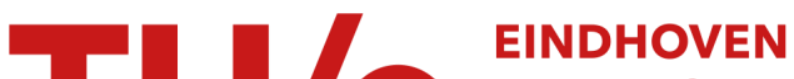 \\ UNIVERSITY OF \\ TECHNOLOGY
}

\section{Large magnetoresistance using hybrid spin filter devices}

\section{Citation for published version (APA):}

LeClair, P. R., Ha, J. K., Swagten, H. J. M., Kohlhepp, J. T., van de Vin, C. H., \& Jonge, de, W. J. M. (2002). Large magnetoresistance using hybrid spin filter devices. Applied Physics Letters, 80(4), 625-627.

https://doi.org/10.1063/1.1436284

DOI:

10.1063/1.1436284

Document status and date:

Published: 01/01/2002

\section{Document Version:}

Publisher's PDF, also known as Version of Record (includes final page, issue and volume numbers)

\section{Please check the document version of this publication:}

- A submitted manuscript is the version of the article upon submission and before peer-review. There can be important differences between the submitted version and the official published version of record. People interested in the research are advised to contact the author for the final version of the publication, or visit the $\mathrm{DOI}$ to the publisher's website.

- The final author version and the galley proof are versions of the publication after peer review.

- The final published version features the final layout of the paper including the volume, issue and page numbers.

Link to publication

\section{General rights}

Copyright and moral rights for the publications made accessible in the public portal are retained by the authors and/or other copyright owners and it is a condition of accessing publications that users recognise and abide by the legal requirements associated with these rights.

- Users may download and print one copy of any publication from the public portal for the purpose of private study or research.

- You may not further distribute the material or use it for any profit-making activity or commercial gain

- You may freely distribute the URL identifying the publication in the public portal.

If the publication is distributed under the terms of Article 25fa of the Dutch Copyright Act, indicated by the "Taverne" license above, please follow below link for the End User Agreement:

www.tue.nl/taverne

Take down policy

If you believe that this document breaches copyright please contact us at:

openaccess@tue.nl

providing details and we will investigate your claim. 


\title{
Large magnetoresistance using hybrid spin filter devices
}

\author{
P. LeClair, ${ }^{\text {a) }}$ J. K. Ha, H. J. M. Swagten, J. T. Kohlhepp, C. H. van de Vin, \\ and W. J. M. de Jonge \\ Department of Applied Physics and COBRA Research Institute, Eindhoven University of Technology, \\ P.O. Box 513, 5600 MB Eindhoven, The Netherlands
}

(Received 6 August 2001; accepted for publication 14 November 2001)

\begin{abstract}
A magnetic "spin filter" tunnel barrier, sandwiched between a nonmagnetic metal and a magnetic metal, is used to create a magnetoresistive tunnel device, somewhat analogous to an optical polarizer-analyzer configuration. The resistance of these trilayer structures depends on the relative magnetization orientation of the spin filter and the ferromagnetic electrode. The spin filtering in this configuration yields a previously unobserved magnetoresistance effect, exceeding 100\%. (C) 2002 American Institute of Physics. [DOI: 10.1063/1.1436284]
\end{abstract}

Spin electronic ("spintronic") devices, ${ }^{1}$ based on utilizing the spin as well as the charge of electrons, open up an entirely new class of electronics. Such devices could include nonvolatile magnetic memories, reprogrammable logic, ${ }^{1}$ and quantum computers. ${ }^{2}$ One thing hampering the development of spin electronic devices so far is the lack of sufficiently polarized (nearing $100 \%$ spin polarization) current sources, for instance, for spin injection into semiconductors ${ }^{3}$ or reprogramable logic. ${ }^{1}$ So-called "half-metallic ferromagnets," fully spin-polarized ferromagnets, would circumvent this problem, ${ }^{4}$ but true half metals have proven extremely difficult to realize in practice. ${ }^{5}$ However, rather than simply using a single nearly perfectly polarized material, the phenomenon of spin filtering may also be exploited to create near $100 \%$ polarization. Here, we propose and demonstrate a different approach, combining spin filter tunnel barriers ${ }^{6}$ and spindependent tunneling, ${ }^{7,8}$ similar to a device proposed by Worledge et al. ${ }^{9}$ The combination of a nonmagnetic electrode with a spin filter tunnel barrier is used to effectively mimic a half-metallic tunneling electrode and achieve nearly $100 \%$ spin polarization. Using this "artificial half-metal" bilayer, we additionally employ a second magnetic electrode, creating a nonmagnetic metal/ferromagnetic insulator/ ferromagnetic metal device. We utilize EuS as the magnetic insulator, with Gd ferromagnetic and Al nonmagnetic electrodes. The tunnel current in this case depends on the relative magnetization orientation of the EuS filter and the Gd "analyzer," in analogy to a half-metallic ferromagnet/insulator/ ferromagnet tunnel junction. The spin filtering in this configuration yields a previously unobserved magnetoresistance effect, which we dub "spin filter injection magnetoresistance" (SFIM), exceeding 100\%, suggesting a filtering efficiency close to $100 \%$. The present scheme would also circumvent impedance mismatch problems with semiconducting counter electrodes, and thus potentially allow spin injection from even a nonmagnetic metal into a semiconductor.

The principle of spin filtering with magnetic semiconductors has been demonstrated in field emission

\footnotetext{
a) Author to whom all correspondence should be addressed; Electronic mail: pleclair@alum.mit.edu
}

experiments, ${ }^{10}$ and in tunnel junctions using a superconducting spin detector. ${ }^{6}$ The concept of spin filtering is illustrated in Fig. 1, using the well-known semiconducting Euchalcogenide, EuS, ${ }^{11,12}$ which is ferromagnetic below $T_{C}$ $\sim 16.8 \mathrm{~K}$, as a spin filter. Above the $T_{C}$ of the EuS barrier, both spin-up and spin-down electrons experience the same potential barrier [Fig. 1(a)]. Below $T_{C}$, due to the spin splitting of the conduction band in EuS (which forms the top of the tunnel barrier), the barrier height becomes spin dependent, as shown in Fig. 1(b). As a result of the exponential dependence of tunnel current on barrier height, one spin channel has a much larger tunneling probability than the other, resulting in a nearly $100 \%$ spin-polarized current.

With a magnetic metal, we must consider the role of the spin-polarized density of states in the electrode as well. The tunnel current depends on the number of filled states in the
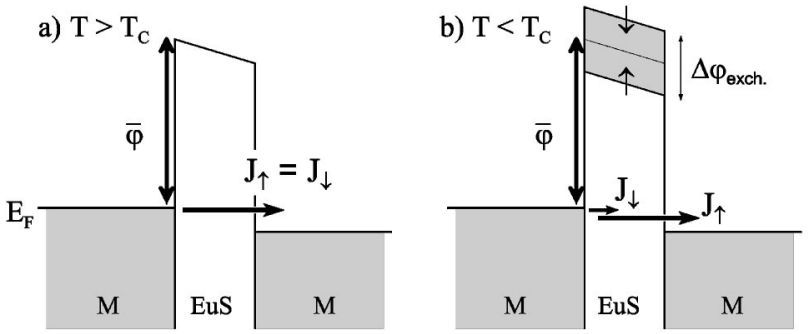

c)

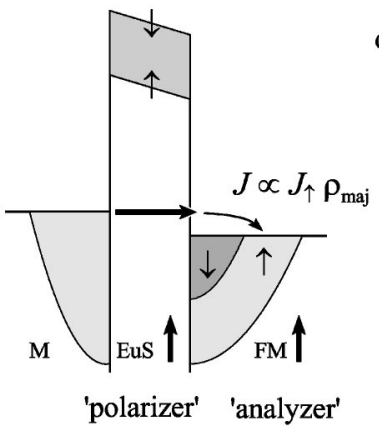

d)

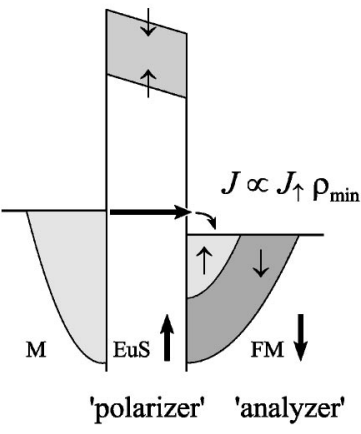

FIG. 1. Schematic illustration of spin filtering and the MR effect. (a), above $T_{C}$ of the EuS filter the two spin currents are equal. (b), below the $T_{C}$ of EuS, the tunnel barrier is spin split, resulting in a highly spin polarized tunnel current. With a ferromagnetic (FM) electrode, the tunnel current depends on the relative magnetization orientation. For parallel alignment $(\mathrm{P})$, (c), a large current results, while for antiparallel alignment (AP), (d), a small current results. 


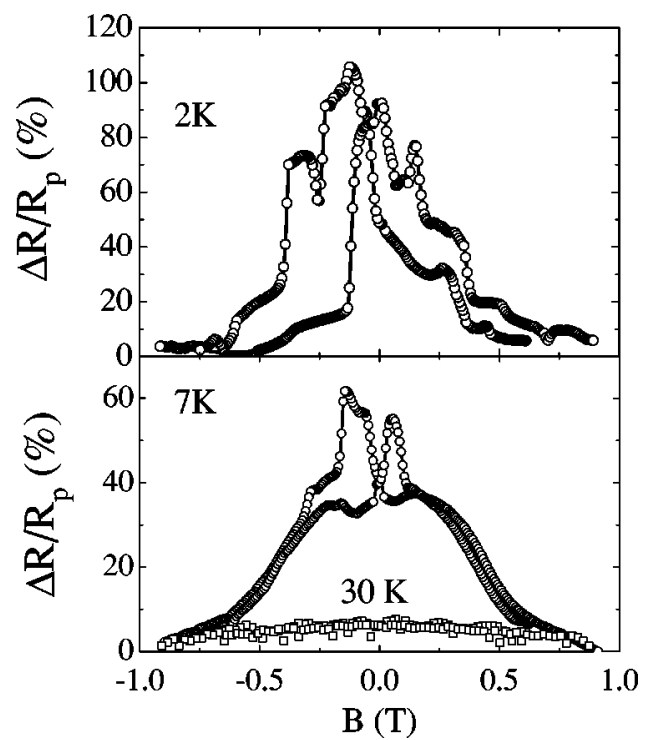

FIG. 2. (a) MR as a function of magnetic field at $2 \mathrm{~K}$ (well below the EuS $T_{C}$ ) and (b) at $7 \mathrm{~K}$, and at $30 \mathrm{~K}$ (well above the EuS $T_{C}$ ).

first electrode as well as the number of available states in the second. Using one magnetic electrode, Fig. 1(c), the density of available states in the magnetic electrode is spin dependent, and the tunnel current will depend on the relative orientation of the filtered spins (i.e., the EuS magnetization direction) and the electrode magnetization. For parallel alignment, Fig. 1(c), only majority (spin up) electrons tunnel through the filter, and thus they can only tunnel into majority states in the magnetic electrode, resulting in a large tunnel current. For the antiparallel case, [Fig. 1(d)], the current is minimal, since only the minority (spin down) states are available in the ferromagnet. One may consider this device analogous to a polarizer/analyzer optical configuration, albeit with a less-than-perfect analyzer, or a magnetic tunnel junction with one half-metallic electrode. The magnitude of the expected SFIM effect may be estimated within a simple twocurrent model, ${ }^{7,13}$ assuming spin conservation in the tunneling process, as $\Delta R / R_{p}=2 P_{m} P_{f} /\left(1-P_{m} P_{f}\right)$, where $P_{m}$ is the spin polarization of the ferromagnetic electrode, $P_{f}$ is the efficiency (polarization) of the spin filter, and $\Delta R / R_{p}$ is the change in resistance between parallel and antiparallel magnetization configurations normalized by the resistance in the parallel state. For a ferromagnetic electrode polarization of $50 \%,{ }^{7}$ and a filter efficiency of $90 \%,{ }^{6}$ one may expect a SFIM effect of more than $160 \%$.

Devices were fabricated using conventional ultrahigh vacuum sputtering techniques with in situ shadow masks onto oxidized $\mathrm{Si}(100)$ wafers. The EuS tunnel barrier was grown at $300^{\circ} \mathrm{C}$, while the metallic layers were grown at ambient temperature. Figure 2 shows resistance versus magnetic field, measured at a dc bias of $\approx 5 \mathrm{mV}$, for a $\mathrm{Si} / \mathrm{SiO}_{2} / \mathrm{Ta} 5 \mathrm{~nm} / \mathrm{Al} 3 \mathrm{~nm} / \mathrm{EuS} 5 \mathrm{~nm} / \mathrm{Gd} 15 \mathrm{~nm}$ structure at 2 $\mathrm{K}$ (well below the $\mathrm{EuS} T_{C}$ ), $30 \mathrm{~K}$ (well above the $\mathrm{EuS} T_{C}$ ), and at $7 \mathrm{~K}\left(T / T_{C} \sim 0.4\right)$. At $2 \mathrm{~K}$, an effect of $\sim 100 \%$ (in some cases more than $130 \%$ ) is observed, clearly indicating the efficiency of the spin filtering. However, at $30 \mathrm{~K}$, above the EuS $T_{C}$, almost no magnetoresistance $(<5 \%)$ is observed, indicating clearly that the observed effects are due to the presence of a ferromagnetic spin filter barrier (the small Downloaded 08 Oct 2009 to 131.155.151.77. Redistribution subject persisting effect is due only to the field-induced magnetization in the EuS layer). Similar effects were observed in many other samples, with magnetoresistance (MR) effects ranging from $30 \%$ or less to $130 \%$. In all cases, the MR was approximately symmetric in bias for low biases. Returning to the 2 $\mathrm{K}$ data, for sufficiently high fields $(\sim 0.5 \mathrm{~T})$, when both magnetizations are parallel a low resistance state is observed. For some small fields, although the magnetization orientation in these structures is not completely well defined, a near antiparallel alignment is reached, and thus, a high resistance state is observed. At $7 \mathrm{~K}$, a large effect is still observed, and the switching behavior is also more controlled, though still a complete antiparallel alignment is not reached. For completely antiparallel alignment, an even larger SFIM effect is anticipated. Given the observed SFIM effects of more than $130 \%$, it can be determined using the aforementioned simple model that for a $\mathrm{Gd}$ polarization of $45 \%,{ }^{14}$ our filter efficiency is $87.5 \%$, in line with previous investigations. ${ }^{6}$ Further, to indicate that a relative parallel or (nearly) antiparallel alignment is possible, we have measured magnetization versus magnetic field at $5 \mathrm{~K}$ for separate EuS $5.0 \mathrm{~nm} / \mathrm{Gd}$ bilayers. Two distinct switching events are observed, one at low field corresponding to the EuS magnetization reversal, and one at higher fields corresponding to the Gd magnetization reversal. At $5 \mathrm{~K}$, the EuS layer switched at approximately 200 Oe, while the Gd layer began to switch at $\sim 800$ Oe and was fully reversed at $\sim 2000 \mathrm{Oe}$, roughly in line with the MR behavior. The presence of two distinct magnetization reversals indicates that the Gd electrode and EuS barrier can be switched independently (with some preliminary evidence for antiferromagnetic coupling between EuS and Gd), and may be aligned parallel or nearly antiparallel. Parenthetically, we note that the additional fine structure in the MR loops may be due to the fact that the MR effect is exponentially sensitive to the EuS magnetization (discussed next), and any instabilities in the EuS magnetization will be exponentially amplified in the MR signal.

Additional evidence of the nature of the spin filtering phenomenon can be obtained from the temperature dependence of the junction resistance. If spin filtering is present, one can expect the junction resistance to decrease as the temperature decreases. ${ }^{6,10-12}$ Specifically, below $T_{C}$, the barrier height for spin-up electrons is much lower (by $\sim 0.18 \mathrm{eV})^{11,12}$ than for either spin-up or spin-down electrons above $T_{C}$. Thus, spin-up electrons preferentially tunnel because of this lowered barrier, leading to a resistance decrease at low temperatures in addition to the high spin polarization. More quantitatively, the tunnel resistance (for a vanishing external bias) can be expressed within a simple free-electron tunneling model ${ }^{6,7,13}$ as

$$
R_{\uparrow \downarrow} \sim \exp \left[-d \varphi_{\uparrow \downarrow}^{1 / 2}(T)\right], \quad \varphi_{\uparrow \downarrow}(T)=\bar{\varphi} \mp J_{d f} S \sigma(T),
$$

where $d$ is the barrier thickness, $T$ is the temperature, $\bar{\varphi}$ is the average tunnel barrier height, $J_{d f}$ is the $d-f$ exchange constant for EuS, ${ }^{11} S=7 / 2$ is the spin quantum number of a $\mathrm{Eu}^{2+}$ ion, $\sigma(T)$ is the reduced magnetization $M(T) / M(T$ $=0)$ of EuS, and the $\uparrow(\downarrow)$ denote spin up(down) electrons. We expect, then, that the temperature dependence of the tunnel resistance should scale (exponentially) with the magnetization of the EuS filter. Shown in Fig. 3 is the normalized to AlP license or copyright; see http://apl.aip.org/apl/copyright.jsp 


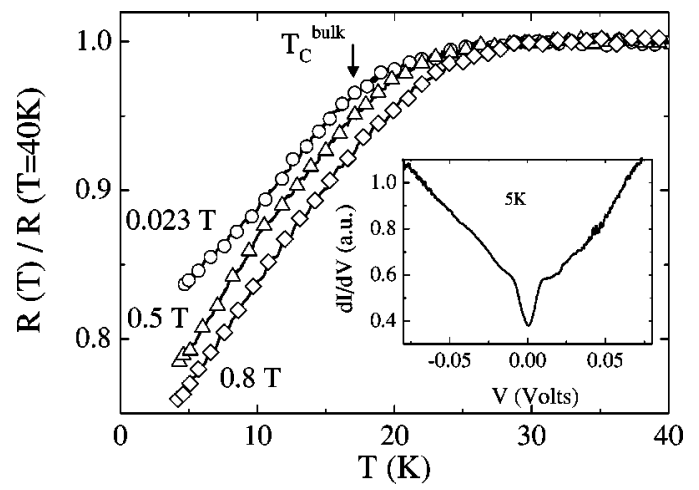

FIG. 3. Normalized resistance vs temperature behavior for several values of magnetic field: $\mathrm{B}=0.0 \mathrm{~T}$ (circles), $\mathrm{B}=0.3 \mathrm{~T}$ (squares), and $\mathrm{B}=0.8 \mathrm{~T}$ (triangles). Inset: $d I / d V-V$ characteristics at $5 \mathrm{~K}$ for a representative $\mathrm{Al} /$ EuS/Gd device.

tunnel resistance (taken at a dc bias of $250 \mathrm{mV}$ ) as a function of temperature, in several applied fields, for an $\mathrm{Al} / \mathrm{EuS} / \mathrm{Gd}$ junction. Indeed, a clear decrease of the tunnel resistance is observed below the $T_{C}$ of EuS, which provides proof that spin filtering is present. Further, the broadening of this resistance transition with increasing applied field is expected from the smearing of the magnetization-temperature behavior near the transition in the presence of an external field. ${ }^{15}$ This clearly indicates that the resistance transition is related to the magnetic phase transition in EuS. Further, the exponential sensitivity of the tunnel resistance to the reduced magnetization of the EuS layer may explain the noise observed in the $R(H)$ data, as small fluctuations in magnetization are amplified in the resistance signal. We note parenthetically that although the absolute resistance of these devices is quite high, as expected for a $50 \AA$ barrier, reducing the EuS thickness by a factor of 2 or more is possible, and would reduce the device resistance dramatically without seriously effecting the magnetic quality. ${ }^{16}$

Finally, to indicate that all of the observed phenomena are genuinely due to tunnel transport, in the inset of Fig. 3 we show a conductance-voltage $(d I / d V-V)$ curve at $5 \mathrm{~K}$ for an $\mathrm{Al} / \mathrm{EuS} / \mathrm{Gd}$ junction. A roughly parabolic and symmetric behavior is observed at higher voltages, indicative of tunnel transport. ${ }^{13}$ At low voltages, a linear contribution is observed, consistent with tunneling assisted by magnon excitations in the Gd layer. ${ }^{17}$ Fitting the current-voltage $(I-V)$ curves to Simmons ${ }^{13}$ model gives barrier heights of $\sim 0.5 \mathrm{eV}$ below the EuS $T_{C}$. By fitting curves below and above $T_{C}$, we obtain an exchange splitting of $\approx 0.36 \mathrm{eV}$ [using Eq. (1)], in agreement with bulk data ${ }^{11,12}$ and previous tunneling measurements. ${ }^{6}$ Though the barrier heights are much lower than previously reported values, ${ }^{6}$ this is probably a result of the defective and polycrystalline nature of our sputtered EuS films. Additionally, at temperatures above the
$\operatorname{EuS} T_{C}$, the tunnel resistance decreases as temperature increases, consistent with of tunnel transport. We may conclude that we are indeed observing magnetoresistance due to spin filtering tunneling via the EuS tunnel barrier.

Summarizing, we report a large magnetoresistance effect in $\mathrm{Al} / \mathrm{EuS} / \mathrm{Gd}$ structures, resulting from the combination of a spin filter tunnel barrier and a ferromagnetic electrode. These ideas may have potential utility for spin injection into semiconductors or for hybrid devices. The large polarization achievable using spin filters, ${ }^{6}$ as well as the lack of any impedance mismatch problem ${ }^{3}$ with semiconductors, makes spin filtering a nearly ideal method for spin injection into semiconductors, enabling alternate spintronic devices. ${ }^{1} \mathrm{Fi}$ nally, a spin-filter operating at room temperature would be possible, utilizing the myriad of ferro- and ferri-magnetic ferrites and garnets, ${ }^{15}$ or potentially the recently predicted room temperature diluted magnetic semiconductors, ${ }^{18}$ which can be made magnetic and insulating above room temperature.

The authors would like to acknowledge J. S. Moodera for helpful discussions. Two of the authors (P.L. and J.K.H.) are supported by The Netherlands Technology Foundation STW, and they also acknowledge support from the Dutch Foundation for Condensed Matter research FOM.

${ }^{1}$ G. A. Prinz, Science 282, 1660 (1998).

${ }^{2}$ D. P. DiVincenzo, Science 270, 255 (1995).

${ }^{3}$ R. Fiederling, M. Keim, G. Reuscher, W. Ossau, G. Schmidt, A. Waag, and L. Molenkamp, Nature (London) 402, 787 (1999).

${ }^{4}$ Y. Ji, G. J. Strijkers, F. Y. Yang, C. L. Chien, J. M. Byers, A. Anguelouch, G. Xiao, and A. Gupta, Phys. Rev. Lett. 86, 5585 (2001).

${ }^{5}$ W. E. Pickett and J. S. Moodera, Phys. Today 5, 39 (2001).

${ }^{6}$ J. Moodera, X. Hao, G. Gibson, and R. Meservey, Phys. Rev. Lett. 61, 637 (1988).

${ }^{7}$ R. Meservey and P. Tedrow, Phys. Rep. 238, 173 (1994).

${ }^{8}$ J. S. Moodera, L. R. Kinder, T. M. Wong, and R. Meservey, Phys. Rev. Lett. 74, 3273 (1995).

${ }^{9}$ D. C. Worledge and T. H. Geballe, J. Appl. Phys. 88, 5277 (2000).

${ }^{10}$ L. Esaki, P. Stiles, and S. von Molnar, Phys. Rev. Lett. 19, 852 (1967).

${ }^{11}$ A. Mauger and C. Godart, Phys. Rep. 141, 51 (1986).

${ }^{12} \mathrm{P}$. Wachter, in Handbook on the Physics and Chemistry of Rare Earths (North-Holland, Amsterdam, 1979), Vol. I, Chap. 19.

${ }^{13}$ J. G. Simmons, J. Appl. Phys. 34, 1793 (1963).

${ }^{14}$ C. H. Kant (unpublished). The Gd tunneling spin polarization is yet unknown with EuS barriers. We believe that the polarization of $14 \%$ for $\mathrm{Al} / \mathrm{Al}_{2} \mathrm{O}_{3} / \mathrm{Gd}$ quoted in Ref. 7 would increase considerably if repeated under ultrahigh vacuum conditions (as reported for Ni, Ref. 7).

${ }^{15}$ R. C. O'Handley, in Modern Magnetic Materials, Principles and Applications (Wiley, New York, 2000).

${ }^{16}$ A. Stachow-Wójcik, T. Story, W. Dobrowolski, M. Arciszewska, R. R. Gałązka, M. W. Kreijveld, C. H. W. Swste, H. J. M. Swagten, W. J. M. de Jonge, A. Twardowski, and A. Y. Sipatov, Phys. Rev. B 60, 15220 (1999).

${ }^{17}$ S. Zhang, P. M. Levy, A. C. Marley, and S. S. P. Parkin, Phys. Rev. Lett. 79, 3744 (1997).

${ }^{18}$ T. Dietl, H. Ohno, F. Matsukura, J. Cibert, and D. Ferrand, Science 287, 1019 (2000). 